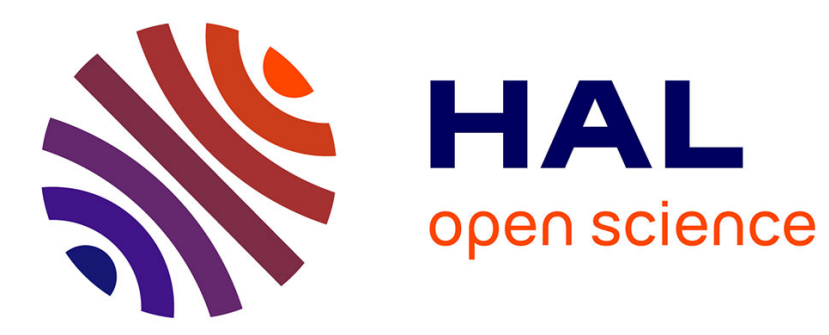

\title{
A sufficient condition for slow decay of a solution to a semilinear parabolic equation
}

Imen Ben Arbi, Alain Haraux

\section{To cite this version:}

Imen Ben Arbi, Alain Haraux. A sufficient condition for slow decay of a solution to a semilinear parabolic equation. 2010. hal-00518113

\section{HAL Id: hal-00518113 \\ https://hal.science/hal-00518113}

Preprint submitted on 17 Sep 2010

HAL is a multi-disciplinary open access archive for the deposit and dissemination of scientific research documents, whether they are published or not. The documents may come from teaching and research institutions in France or abroad, or from public or private research centers.
L'archive ouverte pluridisciplinaire HAL, est destinée au dépôt et à la diffusion de documents scientifiques de niveau recherche, publiés ou non, émanant des établissements d'enseignement et de recherche français ou étrangers, des laboratoires publics ou privés. 


\title{
A sufficient condition for slow decay of a solution to a semilinear parabolic equation
}

\author{
I.Ben Arbi \& A. Haraux \\ 1- UPMC Univ Paris 06, UMR 7598, Laboratoire Jacques-Louis Lions, \\ F-75005, Paris, France. \\ 2- CNRS, UMR 7598, Laboratoire Jacques-Louis Lions, \\ Bô̂te courrier 187, 75252 Paris Cedex 05, France
}

Résumé: On considere l'équation $\psi_{t}-\Delta \psi+c|\psi|^{p-1} \psi=0$ avec les conditions aux limites de Neumann dans un ouvert connexe borné de $\mathbb{R}^{n}$ avec $p>1, c>0$. On montre que si la donnée initiale est petite en norme $L^{\infty}$ et si sa moyenne dépasse en valeur absolue un certain multiple de la puissance $p$ de sa norme $L^{\infty}$, alors $\psi(t, \cdot)$ décroit comme $t^{-\frac{1}{(p-1)}}$.

Abstract: We consider the equation $\psi_{t}-\Delta \psi+c|\psi|^{p-1} \psi=0$ with Neumann boundary conditions in a bounded smooth open connected domain of $\mathbb{R}^{n}$ with $p>1, c>0$. We show that if the initial condition is small enough and if the absolte value of its average overpasses a certain multiple of the $p$ th power of its $L^{\infty}$ norm, then $\psi(t, \cdot)$ decreases like $t^{-\frac{1}{(p-1)}}$.

Keywords: slow decay, parabolic equation 


\section{Introduction and position of the problem.}

In this paper we consider the following nonlinear parabolic equation

$$
\left\{\begin{array}{l}
\psi_{t}-\Delta \psi+g(\psi)=0 \quad \text { in } \quad \mathbb{R}^{+} \times \Omega \\
\frac{\partial \psi}{\partial n}=0 \quad \text { on } \quad \mathbb{R}^{+} \times \partial \Omega
\end{array}\right.
$$

where $\Omega$ is a bounded smooth open connected domain of $\mathbb{R}^{n}$ and $g \in C^{1}(\mathbb{R})$ satisfies

$$
g(0)=0
$$

and for some $p>1$

$$
\exists c>0, \forall s \in \mathbb{R}, \quad 0 \leq g^{\prime}(s) \leq c|s|^{p-1} .
$$

From (1.2)-(1.3)we deduce that $g(s)$ has the sign of $s$ and

$$
\forall s \in \mathbb{R}, \quad|g(s)| \leq \frac{c}{p}|s|^{p}
$$

We define the operator $A$ by

$$
D(A)=\left\{\psi \in H^{2}(\Omega), \frac{\partial \psi}{\partial n}=0 \quad \text { on } \quad \partial \Omega\right\}
$$

and

$$
\forall \psi \in D(A), \quad A \psi=-\Delta \psi
$$

On the other hand the operator $B$ defined by

$$
D(B)=\left\{\psi \in L^{2}(\Omega),-\Delta \psi+g(\psi) \in L^{2}(\Omega) \text { and } \frac{\partial \psi}{\partial n}=0 \quad \text { on } \quad \partial \Omega\right\}
$$

and

$$
\forall \psi \in \text { in } D(B), \quad B \psi=-\Delta \psi+g(\psi)
$$

is well-known to be maximal monotone in $L^{2}(\Omega$. As a consequence of $[2,3]$ for any $\psi_{0} \in L^{2}(\Omega)$ there exists a unique weak solution of the equation

$$
\psi^{\prime}+B \psi=0 \quad \text { on } \quad \mathbb{R}^{+} ; \quad \psi(0, x)=\psi_{0} .
$$

In addition it is well known that if $\psi_{0} \in L^{\infty}(\Omega), \psi(t, \cdot)$ remains in $L^{\infty}(\Omega)$ for all $t>0$. Finally [9] contains an estimate of the solution in $C(\bar{\Omega})$ and $C^{1}(\bar{\Omega})$ for $t>0$, which is valid for any sufficiently regular domain.

We recall two results from [1] 
Theorem 1.1. Let $g$ satisfy (1.2) and (1.3). Then any solution $\psi$ of (1.1) satisfies the following alternative as $t \rightarrow \infty$ : either

$$
\|\psi(t, \cdot)\|_{\infty} \leq C e^{-\lambda_{2} t}
$$

or

$$
\exists c^{\prime}>0, \forall p>1, \forall t \geq 1, \quad \text { such that }\left|\int_{\Omega} \psi(t, x) d x\right| \geq c^{\prime} t^{-\frac{1}{p-1}}
$$

where $\lambda_{2}>0$ is the second eigenvalue of $A$ in $D(A)$.

The proof of Theorem1.1 relies on the following basic fact. Defining the orthogonal projection $P: H \longrightarrow N$, where $H=L^{2}(\Omega)$ and $N=\operatorname{ker}(A)$ is the set of constant functions, we recall

Proposition 1.2. Let $\psi \in C\left(\mathbb{R}^{+}, L^{\infty}\right)$ any solution of (1.1). Assume that $g$ is locally Lipschitz and nondecreasing. Then we have

$$
\|\psi(t)-P \psi(t)\|_{2} \leq\|\psi(0)-P \psi(0)\|_{2} e^{-\lambda_{2} t}
$$

where $\|\cdot\|_{2}$ denotes the norm in $L^{2}(\Omega)$ and $\lambda_{2}>0$ is the second eigenvalue of $-\Delta$ in $L^{2}(\Omega)$ with Neumann boundary conditions .

Finally in [1] the following result was established

Proposition 1.3. Let $g$ satisfy (1.2) and (1.3). Then if $\psi(0, \cdot) \geq 0$ and $\psi(0, \cdot)$ does not vanish a.e in $\Omega$, the solution $\psi$ of (1.1) satisfies (1.7).

It is a natural question, since constant initial data give rise to solutions which satisfy (1.7), to wander what can be said when the initial datum is close to a constant in some sense. One way of expressing proximity to a constant would be to assume that the average is large compared to the projection on the subspace of functions with average 0 . The main result of this paper will show that such a condition actually implies (1.7) at least if $\psi(0)$ is small enough in $L^{\infty}(\Omega)$. Actually our main result is more general and contains the fact that all solutions with constant non-zero initial data satisfy (1.7). 


\section{Main result.}

Theorem 2.1. Let $g$ satisfy (1.2) and (1.3) and $\phi \in L^{\infty}(\Omega)$. Then under the conditions

$$
\begin{gathered}
\|\phi\|_{2 p-2}^{p-1}\|(I-P) \phi\|_{2}<\frac{(p-1)|\Omega|}{2 p^{2}}\left(\frac{p \lambda_{2}}{c}\right)^{\frac{p}{p-1}} \\
|P \phi|>\frac{2 p c}{(p-1) \lambda_{2}|\Omega|}\|\phi\|_{2 p-2}^{p-1}\|(I-P) \phi\|_{2}
\end{gathered}
$$

the solution $\psi$ of (1.1) such that $\psi(0)=\phi$ satisfies

$$
\exists \delta>0, \forall t \geq 1, \quad\left|\int_{\Omega} \psi(t, x) d x\right| \geq \delta t^{-\frac{1}{p-1}}
$$

Proof. Following the notation from [1] we set $\psi=u+w$, where $u=P \psi$ and $w=(I-P) \psi$. By projecting (1.1) on $N$ we have

$$
u^{\prime}+P(g(\psi))=0,
$$

which can be rewritten as

$$
u^{\prime}+g(u)=-P(g(\psi)-g(u))
$$

By the assumption (1.3), we deduce that

$$
|P(g(\psi)-g(u))| \leq \frac{1}{|\Omega|}\|g(\psi)-g(u)\|_{1} \leq \frac{c}{|\Omega|}\left(\|\psi\|_{2 p-2}^{p-1}+\|u\|_{2 p-2}^{p-1}\right)\|w\|_{2} .
$$

By Proposition 1.2 and the fact that $P$ is contractive in all $L^{p}$ spaces we have the estimate

$$
|P(g(\psi)-g(u))| \leq K e^{-\lambda_{2} t}
$$

with

$$
K=\frac{2 c}{|\Omega|}\|\psi(0)\|_{2 p-2}^{p-1}\|w(0)\|_{2}
$$

Assuming, by contradiction, that $\psi$ does not satisfy (1.7), we observe that $u$ is an exponentially decaying solution of

$$
u^{\prime}+g(u)=f(t) \quad \text { in } \quad \mathbb{R}^{+}
$$

where

$$
|f(t)| \leq K e^{-\lambda_{2} t}
$$


It is not difficult to check that such an exponentially decaying solution is unique since the difference of two solutions of this type is either 0 or bounded away from 0 . From this information we shall get an estimate on $u$ by a refinement of the fixed point argument used in [1] . More precisely we shall establish the following

Lemma 2.2. Let $c>0, \gamma>0, p>1$ and $g$ satisfying (1.2) and (1.3) Let $c_{1}>0$ be such that

$$
c_{1}<c_{*}:=\frac{\gamma(p-1)}{p}\left(\frac{p \gamma}{c}\right)^{\frac{1}{p-1}}
$$

then for every function $f$ satisfying

$$
|f(t)| \leq c_{1} e^{-\gamma t}
$$

there exists a unique function $v \in C^{1}\left(\mathbb{R}^{+}\right)$satisfying

$$
\forall t \geq 0, \quad v^{\prime}+g(v)=f(t)
$$

and

$$
\sup _{t \in(0,+\infty)}\left\{e^{\gamma t}|v(t)|\right\} \leq \frac{p c_{1}}{(p-1) \gamma}
$$

Proof. As in [1] we look for a solution of the integral equation

$$
v(t)=-\int_{t}^{+\infty}(f(s)-g(v(s)) d s
$$

in the function space :

$$
X=\left\{v \in C(0,+\infty) ; \sup _{t \in(0,+\infty)} e^{\gamma t}|v(t)| \leq M\right\},
$$

equipped with the distance associated to the norm

$$
\|v\|_{\gamma}=\sup _{t \in(0,+\infty)} e^{\gamma t}|v(t)|
$$

We consider the operator $\mathcal{T}: X \rightarrow C(0,+\infty)$ defined by

$$
\mathcal{T} v(t)=-\int_{t}^{+\infty}(f(s)-g(v(s)) d s
$$


First we will show that under condition (2.4), we can find $M$ in order to achieve $\mathcal{T}(X) \subset X$. Let $v \in X$, then for all $t \geq 0$,

$$
\begin{aligned}
|\mathcal{T} v(t)| & \leq \int_{t}^{+\infty}|f(s)| d s+\int_{t}^{+\infty}|g(v(s))| d s \\
& \leq \int_{t}^{+\infty}|f(s)| d s+\frac{c}{p} \int_{t}^{+\infty}|v(s)|^{p} d s \\
& \leq \frac{c_{1}}{\gamma} e^{-\gamma t}+\frac{c}{p} M^{p} \int_{t}^{+\infty} e^{-p \gamma s} d s \\
& \leq\left(\frac{c_{1}}{\gamma}+\frac{c M^{p}}{p^{2} \gamma}\right) e^{-\gamma t}
\end{aligned}
$$

We just need to check the condition

$$
\frac{c_{1}}{\gamma}+\frac{c M^{p}}{p^{2} \gamma} \leq M
$$

In order for his condition to be fulfilled for some $M>0$ it is clearly neccessary to assume

$$
\frac{c_{1}}{\gamma} \leq \max _{t>0}\left(t-\frac{c t^{p}}{p^{2} \gamma}\right)=\frac{(p-1)}{p}\left(\frac{p \gamma}{c}\right)^{\frac{1}{p-1}}
$$

The corresponding strict inequality is just equivalent to $c_{1}<c^{*}$. On the other hand if we choose

$$
M:=\frac{p c_{1}}{(p-1) \gamma}
$$

we obtain

$$
\begin{aligned}
\frac{c_{1}}{\gamma}+\frac{c M^{p}}{p^{2} \gamma} & =\frac{c_{1}}{\gamma}\left(1+\frac{c}{p^{2}}\left(\frac{p}{(p-1) \gamma}\right)^{p} c_{1}^{(p-1)}\right) \leq \frac{c_{1}}{\gamma}\left(1+\frac{c}{p^{2}}\left(\frac{p}{(p-1) \gamma}\right)^{p} c_{*}^{(p-1)}\right) \\
& =\frac{c_{1}}{\gamma}\left(1+\frac{c}{p^{2}}\left(\frac{p}{(p-1) \gamma}\right)^{p} \frac{p \gamma}{c}\left(\frac{(p-1) \gamma}{p}\right)^{(p-1)}\right)=M
\end{aligned}
$$

Secondly, we prove that $\mathcal{T}$ is a contraction on $X$. In fact, $\forall v, \bar{v} \in X$, for all $t \geq 0$

$$
\begin{aligned}
|\mathcal{T} v(t)-\mathcal{T} \bar{v}(t)| & \leq \int_{t}^{+\infty}|g(v(s))-g(\bar{v}(s))| d s \\
& \leq c M^{p-1} \int_{t}^{+\infty} e^{-p \gamma s} e^{\gamma s}|v(s)-\bar{v}(s)| d s \\
& \leq \frac{c M^{p-1}}{p \gamma}\|v-\bar{v}\|_{\gamma} e^{-\gamma t} .
\end{aligned}
$$


Then we have

$$
|\mathcal{T} v(t)-\mathcal{T} \bar{v}(t)| e^{\gamma t} \leq \frac{c M^{p-1}}{p \gamma}\|v-\bar{v}\|_{\gamma}
$$

Therefore $\forall v, \bar{v} \in X$

$$
\|\mathcal{T} v-\mathcal{T} \bar{v}\|_{\gamma} \leq \frac{c M^{p-1}}{p \gamma}\|v-\bar{v}\|_{\gamma}
$$

But we have

$$
M^{p-1}=\left(\frac{p}{(p-1) \gamma}\right)^{p-1} c_{1}^{p-1}<\left(\frac{p}{(p-1) \gamma}\right)^{p-1}{c_{*}}^{p-1}=\frac{p \gamma}{c}
$$

Thus $\mathcal{T}$ is a strict contraction on the complete metric space $X$ and the result follows from the Banach fixed point theorem.

In order to apply the estimate $(2.7)$ to $u$ we need to assume $K<\frac{\gamma(p-1)}{p}\left(\frac{p \gamma}{c}\right)^{\frac{1}{p-1}}$ where $\gamma=\lambda_{2}$, which reduces to

$$
\|\psi(0)\|_{2 p-2}^{p-1}\|w(0)\|_{2}<\frac{(p-1)|\Omega|}{2 p^{2}}\left(\frac{p \lambda_{2}}{c}\right)^{\frac{p}{p-1}}
$$

hence (2.1). Then $u$, being equal to $v$, satisfies

$$
|u(t)| \leq \frac{2 p c}{(p-1) \lambda_{2}|\Omega|}\|\psi(0)\|_{2 p-2}^{p-1}\|w(0)\|_{2} e^{-\lambda_{2} t}
$$

Making $t=0$ in this inequality we find

$$
|P \phi| \leq \frac{2 p c}{(p-1) \lambda_{2}|\Omega|}\|\phi\|_{2 p-2}^{p-1}\|(I-P) \phi\|_{2}
$$

contradicting the hypothesis.

\section{Applications}

Since the appearance of our main result is a bit involved, we derive a few simple consequences. 
Corollary 3.1. Let $\phi \in L^{\infty}(\Omega)$ be such that

$$
\int_{\Omega} \phi(x) d x \neq 0
$$

For $\varepsilon>0$ small enough the solution of (1.1) with initial condition $\psi(0)=\varepsilon \phi$ satisfies (1.7).

Corollary 3.2. Let $\phi \in L^{\infty}(\Omega)$ be such that

$$
\begin{gathered}
\|\phi\|_{\infty}<\left(\frac{p-1}{2 p^{2}}\right)^{\frac{1}{p}}\left(\frac{p \lambda_{2}}{c}\right)^{\frac{1}{p-1}} \\
|P \phi|>\frac{2 p c}{(p-1) \lambda_{2}|\Omega|^{\frac{1}{2}}}\|\phi\|_{\infty}^{p-1}\|(I-P) \phi\|_{2}
\end{gathered}
$$

Then the solution of (1.1) with initial condition $\psi(0)=\phi$ satisfies (1.7).

Corollary 3.3. Let $\phi \in L^{\infty}(\Omega)$ satisfying (3.1) and

$$
|P \phi|>\frac{2 p c}{(p-1) \lambda_{2}}\|\phi\|_{\infty}^{p}
$$

Then the solution of (1.1) with initial condition $\psi(0)=\phi$ satisfies (1.7).

We close this section by a concrete example

Corollary 3.4. Let $n=1, \Omega=(0, \pi), g(s)=s^{3}$ and $\phi \in L^{\infty}(\Omega)$ be such that

$$
\|\phi\|_{\infty}<3^{-\frac{2}{3}}
$$

and either

$$
|P \phi|>9\|\phi\|_{\infty}^{2}\|(I-P) \phi\|_{\infty}
$$

or

$$
|P \phi|>9\|\phi\|_{\infty}^{3}
$$

Then the solution of (1.1) with initial condition $\psi(0)=\phi$ satisfies (1.7).

Proof. Obvious consequence of the previous corollaries with $c=3$ and $\lambda_{2}=1$. 


\section{References}

[1] I. Benarbi, Rate of decay to 0 of the solutions to a nonlinear parabolic equation, to appear.

[2] P. Bénilan, H. Brézis, Solutions faibles d'équations d'évolution dans les espaces de Hilbert, Annales de l'institut Fourier, 22 no. 2 (1972), p. 311-329.

[3] H.Brézis, Opérateurs maximaux monotones et semi-groupes de contractions dans les espaces de Hilbert.

[4] C. M.Dafermos, Asymptotic behavior of solutions of evolution equations, Nonlinear Evolution Equations, M. G. Crandall Ed, Academic Press, New-York 1978, 103-123.

[5] A. Haraux, Slow and fast decay of solutions to some second order evolution equations, J. Analyse Mathématiques. 95 (2005), 297-321.

[6] A. Haraux, Decay rate of the range component of solutions to some semilinear evolution equations, J. Analyse Mathématiques.13 (2006), 435445.

[7] A. Haraux, M. A. Jendoubi and O. Kavian, Rate of decay to equilibrium in some semilinear parabolic equations, Journal of Evolution equations 3 (2003), 463-484.

[8] A. Haraux, Systèmes dynamiques dissipatifs et applications, Collection R. M. A. 17, Collection dirigé par P. G. Ciarlet et J. L. Lions, Masson, Paris 1991.

[9] A. Haraux, M. Kirane, Estimations $C^{1}$ pour des problèmes paraboliques semi-linéaires, Annales de la faculté des sciences de Toulouse Sér. 5, 5 no. 3-4 (1983), p. 265-280. 